eCommons@AKU

January 2015

\title{
Induced abortion in 30 Chinese provinces in 2013: a cross-sectional survey
}

Shangchun $\mathrm{Wu}$

The National Research Institute for Family Planning

Marleen Temmerman

Aga Khan University, marleen.temmerman@aku.edu

Kun Wang

The National Research Institute for Family Planning

Shuchen Wang

Ghent University

Jiong Li

Aarhus University

See next page for additional authors

Follow this and additional works at: http://ecommons.aku.edu/ eastafrica_fhs_mc_obstet_gynaecol

Part of the Obstetrics and Gynecology Commons

\section{Recommended Citation}

Wu, S., Temmerman, M., Wang, K., Wang, S., Li, J., Zhang, W. (2015). Induced abortion in 30 Chinese provinces in 2013: a crosssectional survey. Lancet, 386(1), 18-18.

Available at: http://ecommons.aku.edu/eastafrica_fhs_mc_obstet_gynaecol/59 
Authors

Shangchun Wu, Marleen Temmerman, Kun Wang, Shuchen Wang, Jiong Li, and Wei-Hong Zhang 


\title{
Induced abortion in 30 Chinese provinces in 2013: a cross-sectional survey
}

\author{
Shangchun Wu, Marleen Temmerman, Kun Wang, Shuchen Wang, Jiong Li, Wei-Hong Zhang, for the INPAC group*
}

Published Online October 30, 2015

*listed at the end of the abstract

The National Research Institute for Family Planning (NRIFP), Beijing, China (Prof SWu MPH, K Wang MPH); International Centre for Reproductive Health (ICRH), Ghent University ، Belgium

(Prof M Temmerman PhD, SWang MPH, W-H Zhang PhD);

The Department of

Reproductive Health and

Research, WHO, Geneva, Switzerland

(Prof M Temmerman); Section for Epidemiology, Department

of Public health, Aarhus

University, Aarhus, Denmark (J Li PhD); and School of Public

Health, Université Libre de

Bruxelles (ULB), Brussels, Belgium (ProfW-H Zhang PhD)

Correspondence to:

Prof Wei-Hong Zhang, International Centre for Reproductive Health (ICRH), WHO Collaborating Centre, Ghent University, 185 UZP114

(6K4), 9000 Ghent, Belgium wzhang@ulb.ac.be

\section{Abstract}

Background Galloping economic growth and reform in China in the past 30 years has led to dramatic social changes. Attitudes towards sex and sexual behaviour have changed, and premarital sex has become more acceptable. The methods of contraception have changed, and the use of highly effective or long-acting contraceptive methods tends to be decreasing, especially in urban areas. Abortion is commonly used to end unintended pregnancy. The aim of this study was to survey the current situation of induced abortions in selected hospitals in 30 provinces in China.

Methods This cross-sectional study was conducted in 295 randomly selected hospitals in 30 Chinese provinces between April and August, 2013. We collected data using a questionnaire filled by the abortion service providers for all women seeking abortion within 12 weeks of pregnancy during a period of two months. The information included self-reported demographic and economic characteristics, history of induced abortion, and use of contraception. The characteristics of women were summarised with counts (percentages) for categorical variables; mean (SD) and range for age of women. All participants signed a written informed consent of which they received a copy. Ethics approvals were obtained from both ethics committees of the National Research Institution for Family Planning (NRIFP), China, and of the Ghent University, Belgium.

Findings 79174 women participated in the study (mean age 28.9 years (SD 1.7; range 13-58), of whom 27134 (35\%) were undergoing a first induced abortion, 28637 (37\%) a second abortion, and $22682(29 \%)$ a third or subsequent abortion. About a third of participants (31\%) were not married and more than half $(61 \%)$ were not local residents. The primary reasons for the unintended pregnancy were contraception failure $(50 \%)$ and non-use of contraception $(44 \%)$.

Interpretation This is the first nationwide large-scale study in $\mathbf{3 0}$ provinces to show that repeated induced abortion is high in China. A family planning programme for young and unmarried people is urgently needed to improve their access to information, advice, and services about contraception and to reduce unintended pregnancies and repeated induced abortion.

Funding The European Commission (EC) under the Seventh Framework Programme (FP7), project number 282490.

Contributors

SWu designed the study, collected data, and drafted the abstract. KW cleared data and commented on the abstract. MT, SWang, and JL commented on the abstract. W-HZ designed the study, and drafted and finalised the abstrcat. All authors have seen and approved the final version of the abstract for publication.

Declaration of interests

We declare no competing interests.

The INPAC group

International Centre of Reproductive Health, Ghent University Belgium: Marleen Temmerman (Consortium Coordinator), Wei-Hong Zhang (Project Leader), Shuchen Wang. Chinese Society of Family Planning - Chinese Medical Association, China: Jian Li, Cheng-Liang Xiong, Li-Nan Cheng, Jun-Li Liu, Qing-Long Meng, Yan Che, Wei-Li Zhao, Hui-Ping Zhang. Fudan University, Shanghai, China: Xu Qian, Ji Liang. National Research Institute for Family Planning, Beijing, China: Shangchun Wu, Yan Zhou, Qing Liu. The Second Affiliated Hospital of Chongqing Medical University, Chongqing, China: Lina Hu, Xiao-Jing Dong, Yi Jiang, Shuai-Bei Liu, Xiao-Ling Gan. Danish Epidemiology Science Centre, University of Aarhus, Aarhus, Denmark: Jørn Olsen, Jiong Li. Liverpool School of Tropical Medicine, Liverpool, UK: Rachel Tolhurst, Susabe-Richards Esther. 